\title{
Constitution of Fibrin-Based Niche for In Vitro Differentiation of Adipose-Derived Mesenchymal Stem Cells to Keratinocytes
}

\author{
Unnikrishnan Sivan, ${ }^{1}$ K. Jayakumar, ${ }^{2}$ and Lissy K. Krishnan ${ }^{1}$
}

\begin{abstract}
Epithelialization of chronic cutaneous wound is troublesome and may require use of skin/cell substitutes. Adipose-derived mesenchymal stem cells (ADMSCs) have immense potential as autologous cell source for treating wounds; they can cross the germ layer boundary of differentiation and regenerate skin. When multipotent adult stem cells are considered for skin regeneration, lineage committed keratinocytes may be beneficial to prevent undesirable post-transplantation outcome. This study hypothesized that ADMSCs may be directed to epidermal lineage in vitro on a specifically designed biomimetic and biodegradable niche. Cells were seeded on the test niche constituted with fibrin, fibronectin, gelatin, hyaluronic acid, laminin V, platelet growth factor, and epidermal growth factor in the presence of cell-specific differentiation medium (DM). The ADMSCs grown on bare tissue culture polystyrene surface in DM is designated DM-control and those grown in basal medium (BM) is the BM-control. Lineage commitment was monitored with keratinocyte-specific markers such as cytokeratin 14, cytokeratin 5, cytokeratin 19, and integrin $\alpha 6$ at the transcriptional/translational level. The in vitro designed biomimetic fibrin composite matrix may have potential application as cell transplantation vehicle.
\end{abstract}

Key words: growth factor; stem cells; tissue engineering; wounds; regeneration

\section{Introduction}

D EEP DERMAL BURNS AND CHRONIC ULCERS are devastating conditions and skin substitutes are considered to be effective for treating such wounds. Advances in tissue engineering technology and fabrication of skin substitute are gaining pace both in regenerative medicine and in cosmetology. A fully constituted nonimmunogenic skin substitute with properties that matches the normal skin is still not achieved. Advances in the field of stem cell biology paved new ways of producing engineered tissue constructs. Among the various potential sources of stem cells, adipose tissue is a preferred choice for harvesting autologous mesenchymal stem cells (MSCs). In 1991, Kaplan correlated ectopic bone formation in subcutaneous tissue and the presence of multipotent stem cells in the corresponding fat. ${ }^{1}$ Since then, differentiation potential of adipose-derived mesenchymal stem cells (ADMSCs) has been widely studied and several types of functional cells generated from this source are explored for treating various diseases. With respect to skin tissue regeneration, many groups investigated strategies for ADMSC application in wound healing. Burn injury has been treated by grafting fat, ${ }^{2}$ and for patients with cutaneous radiation syndrome, ADMSC transplantation has been shown to enhance wound healing significantly. ${ }^{3}$ However, the transplantation of ADMSC is made difficult by their multipotent nature and associated differentiation to lineages such as adipocytes, chondrocytes, or osteocytes. In order to ensure that they cannot produce undesirable cells, transplantation of predifferentiated or lineage-committed cells could be beneficial.

Different strategies are tried out for inducing in vitro lineage commitment of stem cells. Coculture of stem cells with differentiated cells is one such approach; culture of MSCs with mitotically arrested keratinocytes induced lineage commitment of stem cells. ${ }^{4}$ In another study, when human bone marrow (BM) MSCs were cocultured with heat-shocked small airway epithelial cells, fused epitheliallike cells were formed. ${ }^{5}$ Cellular fusion could be also a drawback of coculture experiments. ${ }^{6,7}$

Support from the dermal layer of skin is necessary for formation of normal epithelium. Even when lineage-committed cells from an appropriate source are obtained, transplantation efficiency is limited due to the lack of proper homing niches that favor cell adhesion, proliferation, and differentiation. The application of cells to the wound bed using a carrier

\footnotetext{
${ }^{1}$ Thrombosis Research Unit, Biomedical Technology Wing, ${ }^{2}$ Department of Cardiovascular and Thoracic Surgery, Sree Chitra Tirunal Institute for Medical Sciences and Technology, Trivandrum, Kerala, India.
} 
has been found to be an efficient method in different conditions. Vincent Falanga et al. sprayed BMMSCs embedded in fibrinogen-thrombin mixture on human wounds using a double-barreled applicator and found complete healing of wounds. ${ }^{8}$ Normally, fibrin clot that is formed at the wound site acts as the immediate scaffold for further cell migration, proliferation, and differentiation. ${ }^{9}$ A recent double-blinded, placebo-controlled trial demonstrated that when cells were delivered in fibrin suspension, wounds healed effectively. ${ }^{10}$ Therefore, we hypothesized that fibrin-based matrix immobilized with molecules that have direct involvement in in vivo wound healing may induce ADMSC differentiation to keratinocytes. The aim of this study was to standardize an in vitro niche constituted of insoluble fibrin network into which other adhesive proteins, glycosaminoglycans, and growth factors are immobilized to use as culture matrix, along with keratinocyte-specific differentiation medium (DM) to induce in vitro differentiation of ADMSC to keratinocytes. Differentiation was confirmed using keratinocyte-specific markers, qualitatively and quantitatively at a transcriptional and translational level.

\section{Materials and Methods}

\section{Isolation, culture, and characterization of ADMSCs}

Collection of human adipose tissue was approved by the Institutional Ethics Committee. Tissue samples were obtained from donors 40-60 years old during coronary bypass surgery after getting informed consent; patient details other than age were masked from the laboratory. Isolation of ADMSC was done as described elsewhere. ${ }^{11}$ Briefly, $\sim 10 \mathrm{~g}$ of tissue was incubated with $30 \mathrm{~mL}$ of $1.5 \mathrm{mg} / \mathrm{mL}$ collagenase 1 (Invitrogen) at $37^{\circ} \mathrm{C}$ with continuous shaking for 45-60 min. Enzymatic dissociation of tissue was stopped by the addition of double the volume of serum-containing medium, and the resultant suspension was passed through $180-\mu \mathrm{m}$ nylon mesh (Millipore). Cells were washed by centrifugation. The cell pellet was resuspended in basal medium (BM) consisting of low glucose Dulbecco's modified Eagle's medium (DMEM; Gibco), 10\% fetal bovine serum (FBS; Gibco), and antibiotic/antimycotic (AB/AM) solution (Invitrogen). The cells were seeded on to a $25-\mathrm{cm}^{2}$ tissue culture polystyrene dish (TCPS) and kept at $37^{\circ} \mathrm{C}$ under $5 \% \mathrm{CO}_{2}$. The medium was replenished at $72-\mathrm{h}$ intervals. When $\sim 80 \%$ confluence was reached, cells were passaged by standard trypsinization $(0.05 \%$, Invitrogen) protocol.

Human ADMSCs were characterized using an accepted protocol, and we have already published the data. ${ }^{12}$ Briefly, the plastic-adherent cells after second passage were analyzed with a panel of three positive markers (CD90, CD105, and CD44) and two negative markers (CD34, CD45) by flow cytometry (BD FACS Aria I). For analyzing the flow cytometry data, BD FACS Diva 5.1 version was used. The source of antibodies used for analysis of MSC purity and the markers used for tracking differentiation into keratinocyte is given in Table 1.

\section{Preparation of the matrix}

The tissue culture polystyrene (TCPS) surface was coated with specifically composed biomimetic matrix according to the protocol of Prasad Chennazy and Krishnan. ${ }^{13}$ One milliliter of the composed matrix comprised $5 \mathrm{mg}$ in-house isolated human cryoprecipitate (clottable fibrinogen and fibronectin), $0.2 \%$ gelatin (Sigma), and $100 \mu \mathrm{g}$ hyaluronic acid (in-house purified and characterized ${ }^{14}$ ), $20 \mu \mathrm{g}$ of released platelet growth factor (PGF) prepared per Resmi et al., ${ }^{15} 25 \mu \mathrm{g}$ laminin $\mathrm{V}$ (Sigma), and $250 \mathrm{ng}$ recombinant epidermal growth factor (EGF; Sigma). Briefly, matrix composite was clotted on thrombin adsorbed TCPS to get a thin fibrin layer, and dishes were lyophilized under sterile atmosphere using a freeze drier (Edwards, Modulyo 4K). The TCPS immobilized with composite fibrin matrix were then stored at $4^{\circ} \mathrm{C}$ to $6^{\circ} \mathrm{C}$ until use.

\section{Media composition and controls}

The differentiation medium (DM) comprised DMEM: Ham's F12 (1:1) (Gibco) with 10\% FBS South American origin (Gibco) and $25 \%$ human foreskin derived keratinocyteconditioned medium and growth factor supplements. Human foreskin (obtained with informed consent from a local hospital)derived keratinocytes were isolated using standard protocol. Briefly, with the help of dispase 1, epidermis was separated and single cells were isolated using trypsin and seeded onto the TCPS dishes coated with fibrin composite as described above. Medium from the log phase cultures was aspirated, centrifuged at $1000 \mathrm{~g}$ for $10 \mathrm{~min}$ and resterilized using $0.22-\mu \mathrm{m}$ syringe filters. The conditioned medium was stored

Table 1. List of Antibodies

\begin{tabular}{llll}
\hline Name & Isotype & Source & $\begin{array}{c}\text { Catalog } \\
\text { No. }\end{array}$ \\
\hline CD90 PE conjugated & Mouse IgG & BD Pharmingen & 561970 \\
Endoglin (CD105) PE conjugated & Mouse IgG & Santa Cruz & Ab-18838 \\
CD44 & Rabbit IgG & Abcam & Sb65829 \\
CD34 Alexa Fluor 647 & Mouse IgG & Beckman Coulter & sc-7324 \\
CD45 FITC conjugated & Mouse IgG & Millipore & CBD782 \\
Cytokeratin 14 & Mouse IgG & Santa Cruz & Sc-32721 \\
Cytokeratin 5 & Mouse IgG & Molecular Probes & A21204 \\
Alexa fluor 488 antimouse IgG & Rabbit IgG & Molecular Probes & T7471 \\
Texas Red X Phalloidin & & &
\end{tabular}

FITC, fluorescein isothiocyanate; IgG, immunoglobin G; PE, phycoerythrin. 
at $-20^{\circ} \mathrm{C}$ and used within 2 weeks. The growth factor supplements added into DM were $0.5 \mathrm{ng} / \mathrm{mL}$ of bone morphogenetic protein-4 (BMP-4, R\&D Systems), $5 \mathrm{ng} / \mathrm{mL}$ of EGF (R\&D Systems), $0.4 \mathrm{ng} / \mathrm{mL}$ of hydrocortisone (Sigma), $10 \mu \mathrm{g} / \mathrm{mL}$ of insulin (Sigma), and $1 \times \mathrm{AB} / \mathrm{AM}$ (Gibco). The phenotype of ADMSC grown on test niche (TN), which constituted fibrin-based matrix and DMs, was compared with cells grown on bare TCPS with DM (DM-control) and that of cells grown in bare TCPS with basal medium (BM-control). The same medium composition was used for TN and DM-control to identify the influence of matrix on differentiation of ADMSCs to epidermal keratinocytes. Keratinocytes isolated from human foreskin was used as the positive control to standardize reverse transcriptase polymer chain reactions (RTPCR) for confirming the lineage commitment of ADMSCs. Cells from BM-control were used to set the baseline in quantitative real-time PCR (qRT-PCR) fold-change calculations.

\section{Proliferation assay}

The proliferation of cells was estimated by quantifying the genomic DNA using picogreen assay kit from Molecular Probes, according to the protocol described elsewhere. ${ }^{12}$ Briefly, the cells from TN and DM-control were harvested using standard trypsinization protocol, centrifuged, and the pellet was resuspended in $1 \mathrm{~mL}$ of DNase-free water. The cells were taken through freeze-thaw cycles to ensure complete cell lysis and were incubated with $1 \mathrm{~mL}$ of picogreen working solution, in darkness for $5 \mathrm{~min}$ at $22^{\circ} \mathrm{C}-24^{\circ} \mathrm{C}$. Fluorescence emission was measured at $535-\mathrm{nm}$ wavelength using Qubit 2.0 (Invitrogen).

\section{Characterization of cell phenotype}

For analyzing ADMSC grown on TN, DM-control, and BM-control, cells were harvested by standard trypsin digestion method after 12 days of culture, fixed with $3.7 \%$ formaldehyde, and stained with antibodies for surface antigen such as CD90. To stain intracellular antigens, cells were permeated with $0.2 \%$ triton X-100 for 5 min. After blocking with $0.5 \%$ bovine serum albumin in phosphate-buffered saline, cells were stained using specific antibodies listed in table 1. Stained samples were analyzed by BD FACS Aria I flow cytometer and estimated with BD FACS Diva 5.1 software. Immunofluorescence of cultures were also analyzed, after staining them with antibodies against differentiation marker, using a Leica DMIRB inverted fluorescence microscope equipped with a 1.3 megapixel digital camera. For fluorescence microscopic analysis, the cells were counter stained for actin filaments using Texas red conjugated Phalloidin and overlay pictures were taken using Leica Application Suite (Leica).

The RNA was extracted from cells grown on TN, DMcontrol, and BM-control, after 12 days of culture, using TRIZOL reagent (Invitrogen) according to manufacturer's protocol. Quantitative and qualitative analysis of RNA was carried out using a diode array spectrophotometer (Hewlett Packard 8453). One microgram of total RNA was converted to cDNA using superscript-3 reverse transcriptase enzyme (Invitrogen) using a Master Cycler (Eppendorff). To study the keratinocytes differentiation, qRT-PCR for cytokeratin 5 , cytokeratin 14 , cytokeratin 19 , and integrin $\alpha 6$ genes were analyzed using specific intron spanning primers. Primer

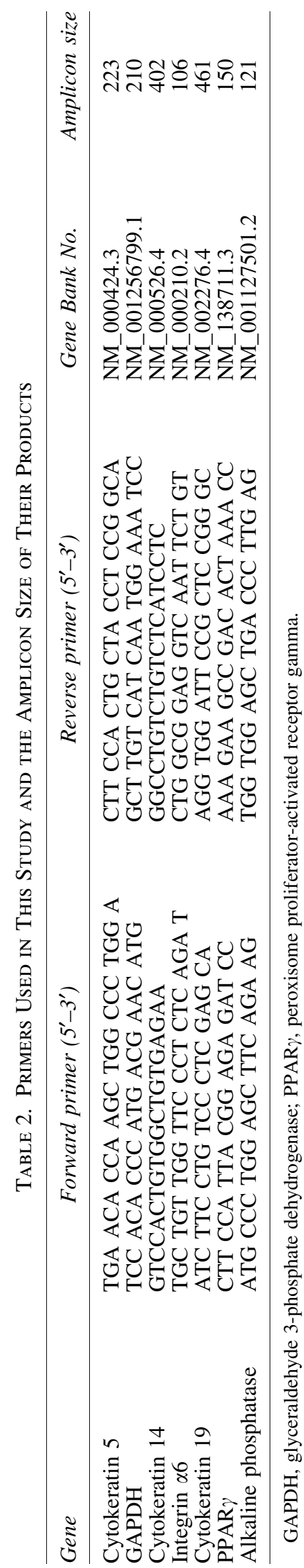


sequence and amplicon size are given in Table 2. To estimate the upregulation of expression, the qRT-PCR was performed using a Chromo4 system (MJ Research). All reactions were carried out for 40 cycles. For each gene, an assessment of quality was performed by examining PCR melt curves after qRT-PCR to ensure product specificity. The cycle threshold $\left(\mathrm{C}_{\mathrm{t}}\right)$ value of the target gene were analyzed after normalization to the $C_{t}$ value of glyceraldehyde 3-phosphate dehydrogenase. Fold change $2^{-\Delta \Delta \mathrm{Ct}}$ was calculated by comparing the expression levels of mRNA extracted from cells of TN, DM-control, and BM-control. Chances of adipogenic, osteogenic, and chondrocyte lineage differentiation were analyzed by RT-PCR. Amplification of genes using specific intron spanning primers for adipocytes (peroxisome proliferator-activated receptor gamma [PPAR $\gamma]$ ), osteocytes (alkaline phosphatase), and chondrocytes (collagen 2) were analyzed by RT-PCR and the product was analyzed by agarose gel electrophoresis.

\section{Statistical analysis}

The mean and standard deviation were calculated for quantitative data and the results are expressed as mean \pm standard deviation of 3-6 replicates. For comparison of quantitative results, significance of difference was detected using students $t$-test; $p<0.05$ was considered statistically significant and denoted with an asterisk $(*)$ in graphical representations (Microsoft Excel).

\section{Results}

Well-characterized pure ADMSC population was used to induce differentiation into keratinocytes. Matrix-directed differentiation of ADMSC to keratinocytes is evident from morphological feature, immunocytochemistry, and mRNA expression analysis. Even in the absence of matrix, the cells grown on bare polystyrene surface were also induced to keratinocytes by the constituents in DM, but the influence of matrix was prominent on the extent of marker expression and survival.

\section{Immunochemical and morphological characterization of cells}

Cells isolated from adipose tissue for a previous study were used for differentiation into keratinocytes also. ${ }^{12}$ All three positive markers-CD90, CD105, and CD44-were expressed in more than $95 \%$ of ADMSCs, while less than $5 \%$ of cells expressed the negative markers CD34 and CD45. After 12 days of culture, only $\sim 15 \%$ of the cells harvested from TN were positive for CD90, but in the BM-control, 90\% cells showed CD90 expression (Fig. 1). On the other hand in the $\mathrm{DM}$-control, there was a minor reduction in $\mathrm{CD} 90^{+}$cells (i.e., only $\sim 80 \%$ cells were $\mathrm{CD} 90^{+}$). The changes in MSC marker expression on the cells during the culture period suggested influence of niche composition; more prominent was the effect of matrix components. Loss of stem-ness is not a proof for differentiation into keratinocytes, but it is a preliminary indication that some lineage commitment has taken place in cells grown on the TN.

Groups of cells were found to transform into hexagonal morphology in $\mathrm{TN}$, which is a characteristic feature of keratinocytes (Fig. 2B). Signs of differentiation were seen in

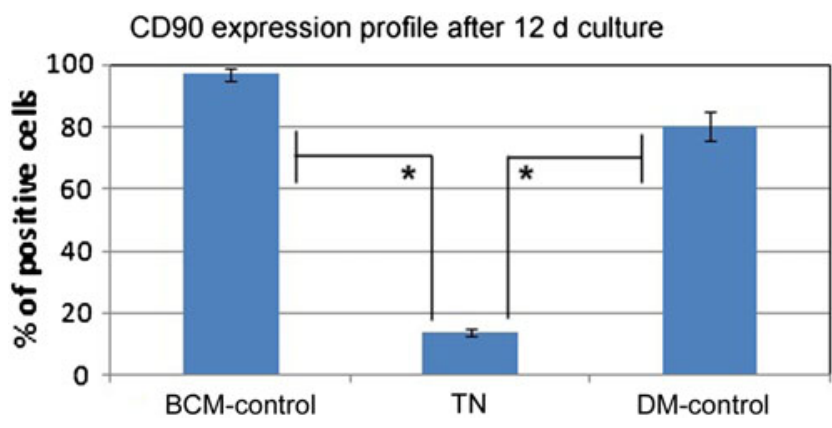

FIG. 1. Stem cell marker expression in adipose-derived mesenchymal stem cells (ADMSCs) before and after differentiation. Expression of CD90 in basal medium (BM)-control, test niche (TN), and differentiation medium (DM)-control were analyzed using a flow cytometer. $* p<0.05$; change in the profile as compared with ADMSC (BM-Control) is statistically significant.

the DM-control also (Fig. 2A). However, it was evident from the low magnification fields that the frequency of keratinocyte-like colonies was higher in the TN (Fig. 2C), suggesting the significant role played by the matrix for inducing differentiation. Typical epithelial cell polarity could be visualized in the cell colonies grown on TN with tight cellular junctions (Fig. 2B). Such signs of differentiation into keratinocytes were observed in TN and DM-control from the eighth day of starting culture on the respective niche. Since there was donor-to-donor variability in the time point at which appearance of keratinocyte-like colonies began, the 12th day of culture on the niche was selected for termination of the experiment and phenotype characterization. When cells were cultured beyond 17 days, differentiated cells grew bigger in size and covered more surface area in TN (Fig. 2D).

\section{Marker expression at transcriptional level}

Undesirable differentiation of ADMSCs into the classical trilineage was ruled out using RT-PCR and subsequent agarose gel electrophoresis. The expression of genes PPAR $\gamma$, alkaline phosphatase (ALP), and collagen 2 ( $\mathrm{Col} 2$ ) specific for adipogenic, osteogenic, and chondrogenic lineages, respectively, were absent in cultures on both DM-control and $\mathrm{TN}$; there was no detectable level of PCR product even after 40 cycles of reaction in each case (Fig. 3).

The mRNA expression profile of three cytokeratin genes (CK5, CK14, and CK19) and the cell adhesion molecule (integrin $\alpha 6$ ) was studied using qRT-PCR. There was a statistically significant fold increase over baseline expression (Fig. 4). Based on the expression level in BM-control, cells from TN showed maximum upregulation of cytokeratin 19 with fold change of $\sim 13$ in qRT-PCR. All the studied genes except integrin $\alpha 6$ in DM-control showed a statistically significant difference over the baseline (BM-control) with a $p<0.05$ when grown in TN and DM-control.

\section{Marker expression at translational level}

Quantitative flow cytometric analysis for cells that expressed $\mathrm{CK} 5^{+} / \mathrm{CK} 14^{+}$showed different level of antigen 

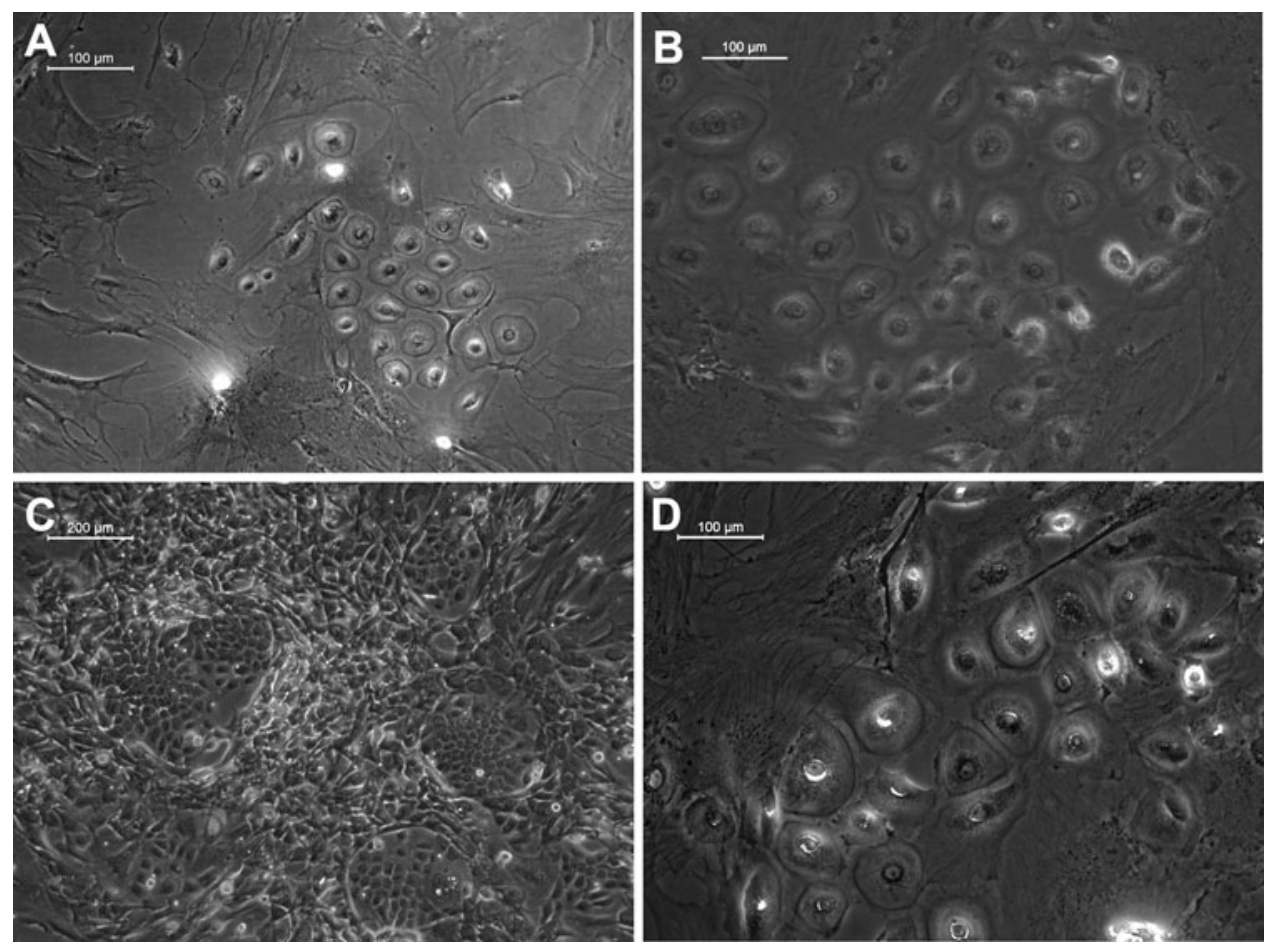

FIG. 2. Morphology of keratinocytes differentiated from ADMSCs. (A) Representative light micrograph of a field from DM-control; (B) Micrograph from TN showing epithelial polarity maintained by cells, which was evident by the convex shape of cells and tight cellular junctions; (C) Micrograph of a larger field of test culture showing more colonies of keratinocyte-like cell islands. (D) Larger cells formed during extended days of culture in test niche. A, B, and D, scale bar $=100 \mu \mathrm{m}$. C, scale $\mathrm{bar}=200 \mu \mathrm{m}$. positivity. The data is presented in Fig. 5, which shows that an average of $40 \%$ cells were $\mathrm{CK} 14^{+}$and $\sim 20 \%$ were $\mathrm{CK} 5{ }^{+}$. When the cells were grown on $\mathrm{TN}$, the numbers of positive cells were significantly higher than that in DMcontrol with $p<0.05$ (Fig. 5). The next step was to confirm

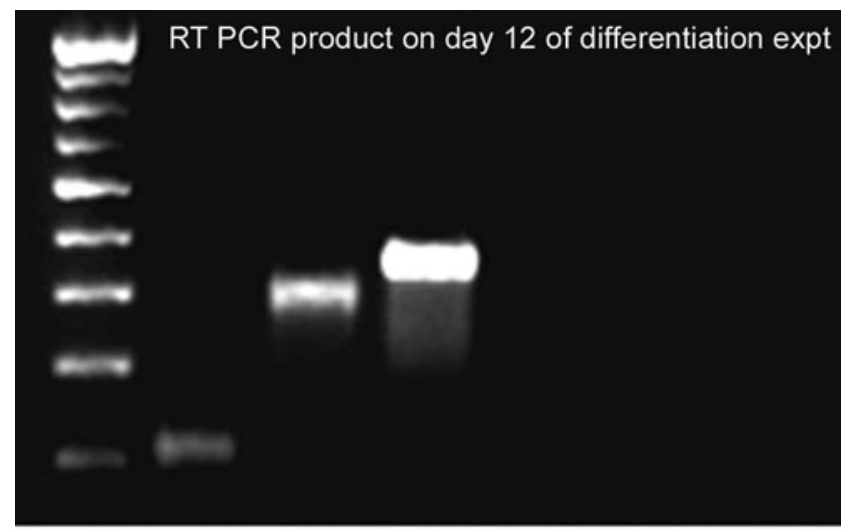

Ladder GAPDH CK14 CK19 Alk P PPARy Col II

FIG. 3. Analysis of reverse transcriptase polymer chain reactions (RT-PCR) products by agarose gel electrophoresis. Cytokeratin 14 (CK14) and CK19 are the positive indicators of MSC differentiation into keratinocytes; negative lanes are for peroxisome proliferator-activated receptor gamma (PPAR $\gamma)$, alkaline phosphatase (ALP), and collagen 2 (Col 2 ), which indicate that ADMSC differentiation into other mesodermal cells such as adipocytes, osteocytes, and chondrocytes, respectively, was absent. Positive controls were used to ensure authenticity of reaction using RNA extracted from osteocyte (cell line) and adipocytes (primary cells) (data not shown). Glyceraldehyde 3-phosphate dehydrogenase (GAPDH) was used as housekeeping gene. Ladder (left lane) ranging from 200 to 1000 base pairs was used to confirm product specificity. keratinocyte-specific markers using immuno-fluorescence microscopy on 12th day of culture. Representative images of CK14 and CK5 stained cells, which were counterstained with actin, are shown in Fig. 6. The differentiated cells formed a tight colony and were surrounded by fibroblastlike population in which only the actin was visible. The cells that bordered keratinocyte-like colonies showed multilayered hilly appearance of fibroblasts as well.

\section{Proliferation}

Cells cultured on DM-control and TN for specific periods were assayed for total DNA by picogreen assay. There was comparable rate of proliferation for the first 7 days in DM-

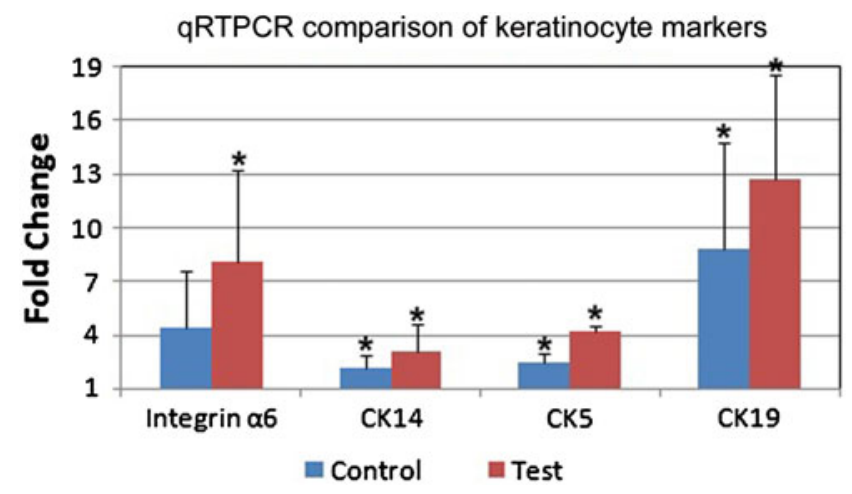

FIG. 4. Real-time PCR (qRT-PCR) analysis data. Four keratinocyte-specific genes were analyzed. GAPDH was used as the internal control and the relative gene expression was calculated using $2^{-\Delta \Delta \mathrm{Ct}}$. Cells cultured on an uncoated tissue culture polystyrene dish without differentiation media were used as the calibrator. ${ }^{*} p<0.05$ relative to the calibrator (BM-control) and between the control and test. 


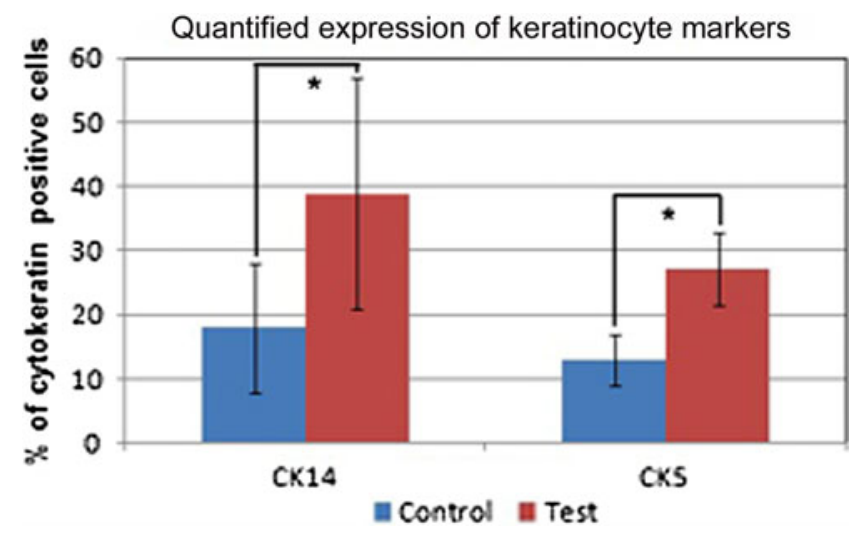

FIG. 5. Quantification of cytokeratin positive cells. CK14 and CK5 positivity in cells after 12 days of culture were analyzed using flow cytometry and the results expressed as a percentage of positive cells. $* p<0.05$.

control and TN. However, more cells were seen in TN by day 7, as compared with DM-control, which may be attributed to the presence of fibrin matrix, which favors growth of all types of cells (Fig. 7). Subsequently, there was only small increase of cells in TN but difference in DNA content between 7 days and 12 days was prominent and significant in DM-
Total DNA in cells during differentiation experiment

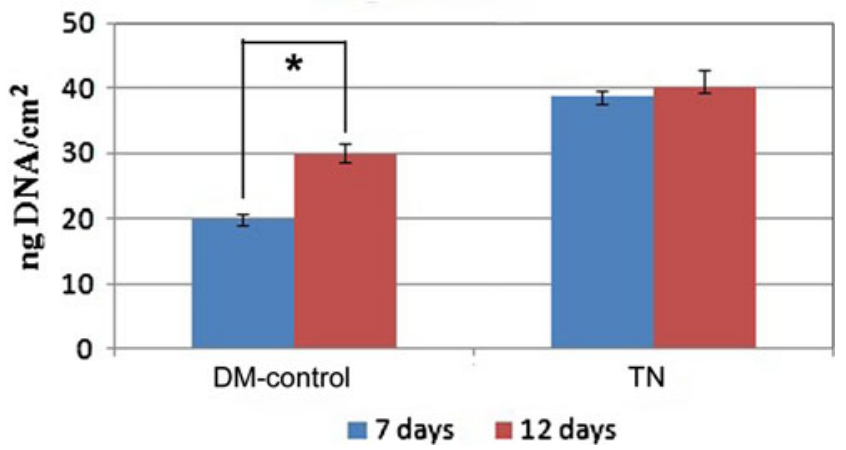

FIG. 7. Total DNA estimation data. Cells cultured on DMcontrol and TN for 7 and 12 days were isolated and lysed. DNA quantity was quantified using the picogreen reagent by fluorescence measurement with Q-bit and expressed as $\mathrm{ng} / \mathrm{cm}^{2} . * p<0.05$.

control. This is noteworthy and substantiates the finding that, cells in TN were lineage committed by seventh day. It is well accepted that once differentiated, cell proliferation slows down. In DM-control, fewer cells differentiated into keratinocytes and probably the remaining ADMSC continued multiplication.
FIG. 6. Cytokeratin positive cells from test culture. (A) cytokeratin 5 (CK5) stained cells; (B) $\mathrm{CK} 5^{+}$cells stained with phalloidin for actin; (C) merged picture of $A$ and $B$. (D) cytokeratin 14 (CK14) stained cells; (E) $\mathrm{CK} 14^{+}$cells stained with phalloidin for actin; (F) merged picture of $D$ and $E$. Arrows indicate the multilayered appearance of undifferentiated cells. The symbol $(+)$ indicates faint CK5 staining and $(+++)$ points more prominent staining.
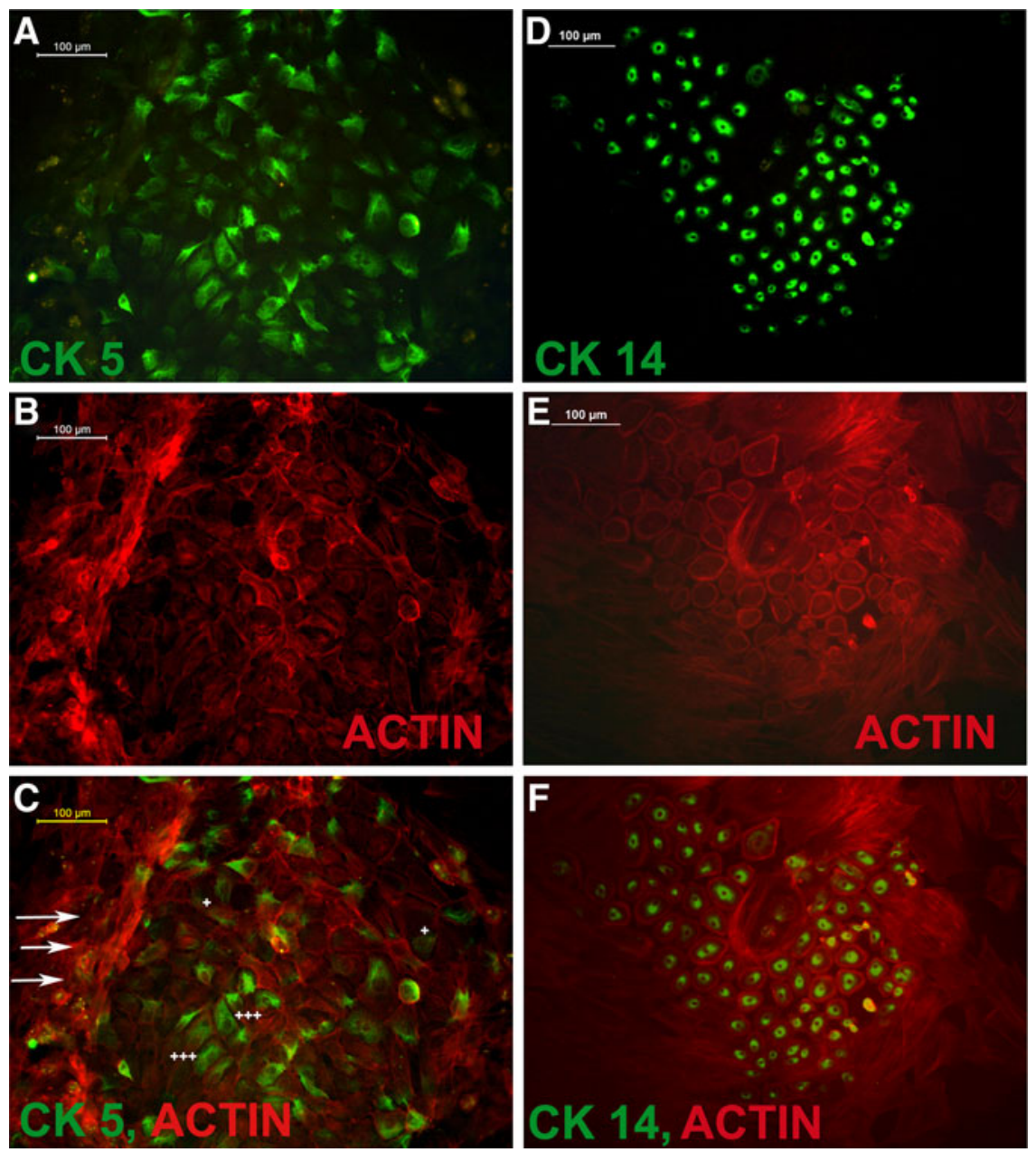


\section{Discussion}

Re-epithelialization is an important step in wound healing. In normal healing, epithelium would grow from the existing stem cells present within skin appendages or from the epithelial precursors present in the wound edges. During a full thickness injury like a burn or in chronic wounds such as diabetic ulcers, skin stem cells may not be available in the local cell pool, causing hindered or absent re-epithelialization. Split thickness skin grafting is considered to be the gold standard in such cases, but the lack of donor site in the case of extended burn or an additional morbid site are limitation of this procedure. Therefore, a possible option is to apply the principles of regenerative medicine, preferably using autologous cells. The use of lineage-committed epidermal cells, originating from ADMSC may produce better outcome. In this study, we attempted to standardize an in vitro niche for differentiation of ADMSCs to skin keratinocytes using cell-specific biomimetic matrix and DM, which constituted the TN. For in vitro construction of the niche, the components chosen were from group of molecules, which are normally seen in in vivo wound healing microenvironments. The biodegradable and biocompatible nature of the niche may qualify its potential use as a carrier for cell transplantation as well. It has been well established that immobilized growth factors are more efficient and long lasting as compared to mobile factors in solution. ${ }^{16,17}$ Therefore, in this study the influence of the growth factors such as EGF and PGF together with cell adhesion molecules like laminin5, fibronectin, gelatin, and hyaluronic acid that were immobilized within the insoluble fibrin network was analyzed. This approach was found successful in differentiating the ADMSCs to keratinocyte lineage with better cell survival. A subpopulation of cultured human ADMSCs retained the fibroblastic morphology and multilayered growth, while another subpopulation had assumed a keratinocyte-like polygonal morphology and expressed epithelial cell markers; cytokeratin $5,14,19$, and integrin $\alpha 6$. Continued culture (17 days) of the differentiated keratinocytes under the same conditions resulted in the formation of cells with larger size, which suggests a process similar to spinous keratinocyte formation. ${ }^{8}$

Mesenchymal stem cells are known to enhance wound healing. Mikako Sasaki et al. (2008) have reported that MSCs may be recruited to the wounded tissue to enhance wound healing by differentiating into keratinocytes, endothelial cells, and pericytes. ${ }^{19}$ They have achieved one cytokeratin positive cell per high power objective field $(0.14 \%)$ when MSCs were injected into an in vivo wound healing site. They reported that when $0.5 \mathrm{nM}$ BMP-4 was added along with keratinocyte basal medium containing $0.3 \mathrm{mM}$ ascorbic acid, $0.5 \mathrm{mg} / \mathrm{mL}$ hydrocortisone, and $3 \mathrm{ng} / \mathrm{mL}$ human epidermal growth factor, the differentiation was enhanced up to $0.48 \%$ during in vitro culture. In our study, we have added a similar concentration of BMP-4 into the media. The TN, which comprised the composite matrix and media, resulted in about $40 \%$ differentiated keratinocytes, which is a significant achievement as compared with other reported results. Since we used a composite niche, factors other than BMP-4 may have contributed to the induction of differentiation. Other than the immobilized components, the constituents added in the culture medium also influenced ADMSC differentiation to keratinocytes as seen in DM-control. However, the extent of differentiation and cell survival was significantly higher when the matrix was present.

Our results show that the expression levels of each cytokeratin gene is different from the others; the mean fold increase of CK5 was 4.22, CK14 was 3.01, and the fold increase was maximum $(\sim 13)$ for CK19. Differential expression of cytokeratins in basal layer keratinocytes and later during their differentiation pathway has been well described. ${ }^{20}$ Relative expression of CK19 was found to be maximal when Hongbin Li et al. (2012) attempted differentiation of rabbit ADMSC to epidermal lineage cells. ${ }^{21}$ They cultured rabbit adipose-derived stem cells with low glucose DMEM supplemented with $2 \% \mathrm{FBS}, 2.5 \mathrm{mM}$ all trans-retinoic acid (ATRA), $20 \mathrm{ng} / \mathrm{mL}$ EGF, $10 \mathrm{ng} / \mathrm{mL}$ hepatocyte growth factor (HGF), $10 \mathrm{ng} / \mathrm{mL} \mathrm{KGF}$, and $0.5 \mathrm{mg} / \mathrm{mL}$ hydrocortisone under air liquid interphase culture. In the current study, other than the CK5/CK14/CK19 expressions, formation of epithelial cell polarity was the most striking observation, which proved the success of our method over air liquid interphase culture.

In a wound-healing environment, MSCs may enhance healing by a variety of means. ${ }^{22}$ The MSCs can modulate the microenvironment by secreting chemokines, act as an immunomodulator, can induce the indigenous stem cells, enhance angiogenesis, and undergo differentiation by themselves. ${ }^{23}$ Optimum growth and expansion of primary keratinocytes normally requires fibroblast feeder layers, ${ }^{24}$ because terminally differentiated cells seldom proliferate. ${ }^{18} \mathrm{We}$ propose that a subpopulation of undifferentiated ADMSC in our culture may be playing the role of feeder cells to support keratinocyte growth and differentiation. The result of proliferation assay is not suggestive of multiplication of differentiated keratinocyte. Additional studies with dual staining for a keratinocyte lineage marker and proliferation marker may be required to make sure if lineage committed cells undergo proliferation or not. Since proliferation of cells in TN did not progress in 7- to 12-day period, and because the lineage commitment in $\mathrm{TN}$ was indicated on eighth day itself, it may be concluded that the differentiated keratinocytes have not proliferated.

Previously, it has been reported that fibrin-based cell delivery systems enhance wound healing. ${ }^{10}$ Here we have used fibrin to construct an in vitro niche, a mimic of in vivo provisional matrix, which is formed immediately after injury to play a dual role of supporting hemostasis and wound healing. Fibronectin associated with fibrin facilitates immobilization of various growth factors and cell adhesion molecules within the fibrin network. Fibronectin, which accelerates cell attachment and growth, could be responsible for the better survival of differentiated cells seen in this study. Lyophilized fibrin discs has been demonstrated to enhance wound healing, so the matrix components that we have put together in this study may be used as a vehicle to transplant cells as well. There is scope for incorporating various antimicrobial agents in to the matrix as well. ${ }^{25}$ The composed matrix, when coated on synthetic polymeric scaffolds, may enhance the cell adhesion and growth to a greater extent. ${ }^{26}$ Therefore, hybrid scaffolds with better handling properties for cell transplantation may be fabricated using the fibrin composite and degradable polymer. 


\section{Summary}

Using an in vitro designed niche, human ADMSCs are induced to differentiate toward epidermal keratinocytes. The differentiated cells showed keratinocyte-specific morphology, epithelial polarity, and expressed common epithelial markers. The biomimetic matrix standardized in this study has potential applications for transplantation of stem cells for regenerative medicine. The study results indicate that autologous ADMSC may be induced to epithelial cells and the standardized strategy may be suitable for translation of the method to clinical practice.

\section{Acknowledgments}

The authors acknowledge Dr. K. Radhakrishnan, Director of Sree Chitra Tirunal Institute for Medical Sciences and Technology (SCTIMST), Trivandrum, and Dr. G.S. Bhuvaneshwar, Head of the Biomedical Technology Wing, SCTIMST, for their valuable support. Authors would like to acknowledge the Kerala State Council for Science, Technology, and Environment, Government of Kerala State, India; HLL Lifecare Limited, Trivandrum; and the Department of Science and Technology (DST), Government of India, for funding the ongoing stem cell research. We would like to acknowledge the fellowship support from DST, Government of India, to U. Sivan.

\section{Author Disclosure Statement}

No competing financial interests exist.

\section{References}

1. Kaplan FS, Hahn GV, Zasloff MA. Heterotopic ossification: two rare forms and what they can teach us. J Am Acad Orthop Surg. 1994;2:288-296.

2. Sultan SM, Barr JS, Butala P, et al. Fat grafting accelerates revascularisation and decreases fibrosis following thermal injury. JPlast Reconstr Aesthet Surg. 2012;65:219-227.

3. Forcheron F, Agay D, Scherthan H, et al. Autologous adipocyte derived stem cells favour healing in a minipig model of cutaneous radiation syndrome. PLoS One. 2012;7:e31694.

4. Sivamani RK, Schwartz MP, Anseth KS, Isseroff RR. Keratinocyte proximity and contact can play a significant role in determining mesenchymal stem cell fate in human tissue. FASEB J. 2011;25:122-131.

5. Spees JL, Olson SD, Ylostalo J, et al. Differentiation, cell fusion, and nuclear fusion during ex vivo repair of epithelium by human adult stem cells from bone marrow stroma. PNAS. 2003;100:2397-2402.

6. Ferrand J, Noël D, Lehours P, et al. Human bone marrowderived stem cells acquire epithelial characteristics through fusion with gastrointestinal epithelial cells. PLoS One. 2011; 6:e29007. DOI: 10.1371/journal.pone.0029007.

7. Garbade J, Schubert A, Rastan AJ, et al. Fusion of bone marrow-derived stem cells with cardiomyocytes in a heterologous in vitro model. Eur J Cardiothorac Surg. 2005;28:685-691.

8. Falanga V, Iwamoto S, Chartier M, et al. Autologous bone marrow-derived cultured mesenchymal stem cells delivered in a fibrin spray accelerate healing in murine and human cutaneous wounds. Tissue Eng. 2007;13:1299-1312.

9. Metcalfe AD, Ferguson MWJ. Tissue engineering of replacement skin: the crossroads of biomaterials, wound healing, embryonic development, stem cells and regeneration. J R Soc Interface. 2007;4:413-437.
10. Kirsner RS, Marston WA, Snyder RJ, Lee TD, Cargill DI, Slade HB. Spray-applied cell therapy with human allogeneic fibroblasts and keratinocytes for the treatment of chronic venous leg ulcers: a phase 2 , multicentre, doubleblind, randomised, placebo-controlled trial. The Lancet 2012;380:977-985.

11. Zhu Y, Liu T, Song K, Fan X, Ma X, Cui Z. Adiposederived stem cell: a better stem cell than BMSC. Cell Biochem Funct. 2008;26:664-75.

12. Unnikrishnan S, Jaykumar K, Krishnan LK. Matrix-directed differentiation of human adipose derived mesenchymal stem cells to dermal-like fibroblasts that produce extracellular matrix. J Tissue Eng Regen Med. 2014. [Epub ahead of print]; DOI: 10.1002/term.1865.

13. Prasad Chennazhy K, Krishnan LK. Effect of passage number and matrix characteristics on differentiation of endothelial cells cultured for tissue engineering. Biomaterials 2005;26:5658-5667.

14. Anilkumar TV, Muhamed J, Jose A, Jyothi A, Mohanan PV, Krishnan LK. Advantages of hyaluronic acid as a component of fibrin sheet for care of acute wound. Biologicals. 2011;39:81-88.

15. Resmi KR, Krishnan LK. Protease action and generation of beta-thromboglobulin-like protein followed by platelet activation. Thromb Res. 2002;107:23-29.

16. Lutolf MP, Gilbert PM, Blau HM. Designing materials to direct stem-cell fate. Nature 2009;462:433-441.

17. Nur-E-Kamal A, Ahmed I, Kamal J, Babu AN, Schindler M, Meiners S. Covalently attached FGF-2 to three-dimensional polyamide nanofibrillar surfaces demonstrates enhanced biological stability and activity. Mol Cell Biochem. 2008;309: 157-166.

18. McGrath JA, Eady R a. J, Pope FM. Anatomy and organization of human skin. In: Rook's Textbook of Dermatology, 7th ed. Burns T, Breathnach S, Cox N, Griffiths C (eds.) Blackwell Publishing, Inc.: Malden, MA; pp. 45-128; 2008.

19. Sasaki M, Abe R, Fujita Y, Ando S, Inokuma D, Shimizu H. Mesenchymal stem cells are recruited into wounded skin and contribute to wound repair by transdifferentiation into multiple skin cell type. J Immunol. 2008;180:25812587.

20. Moll R, Divo M, Langbein L. The human keratins: biology and pathology. Histochem Cell Biol 2008;129:705-733,

21. Li H, Xu Y, Fu Q, Li C. Effects of multiple agents on epithelial differentiation of rabbit adipose-derived stem cells in 3D culture. Tissue Eng Part A. 2012;18:1760-1770.

22. Chen L, Tredget EE, Wu PYG, Wu Y. Paracrine factors of mesenchymal stem cells recruit macrophages and endothelial lineage cells and enhance wound healing. PLoS One. 2008;3:e1886.

23. Kim S-W, Zhang H-Z, Guo L, Kim J-M, Kim MH. Amniotic mesenchymal stem cells enhance wound healing in diabetic NOD/SCID mice through high angiogenic and engraftment capabilities. PLoS One. 2012;7:e41105.

24. Rheinwald JG, Green H. Serial cultivation of strains of human epidermal keratinocytes: the formation of keratinizing colonies from single cells. Cell. 1975;6:331-343.

25. Ragaseema VM, Unnikrishnan S, Kalliyana Krishnan V, Krishnan LK. The antithrombotic and antimicrobial properties of PEG-protected silver nanoparticle coated surfaces. Biomaterials. 2012;33:3083-3092.

26. Pankajakshan D, Philipose LP, Palakkal M, Krishnan K, Krishnan LK. Development of a fibrin composite-coated poly(epsilon-caprolactone) scaffold for potential vascular 
tissue engineering applications. J Biomed Mater Res Part B Appl Biomater. 2008;87:570-579.

Address correspondence to: Lissy K. Krishnan, PhD Thrombosis Research Unit Biomedical Technology Wing Sree Chitra Tirunal Institute for Medical Sciences and Technology Trivandrum-695012 India

E-mail: lissykk@sctimst.ac.in

\section{Abbreviations Used}

$\mathrm{AB} / \mathrm{AM}=$ antibiotic/antimycotic

ADMSCs = Adipose-derived mesenchymal stem cells $\mathrm{BM}=$ basal medium

BPM-4 = bone morphogenetic protein-4 $\mathrm{DM}=$ differentiation medium

DMEM $=$ Dulbecco's modified Eagle's medium

$\mathrm{FBS}=$ fetal bovine serum

MSCs $=$ mesenchymal stem cells

RT-PCR $=$ reverse transcriptase polymer chain reactions TCPS $=$ tissue culture polystyrene $\mathrm{TN}=$ test niche 